\title{
The Effects of Nurse Staffing on Adverse Events, Morbidity, Mortality, and Medical Costs
}

\author{
Sung-Hyun Cho $\boldsymbol{\nabla}$ Shaké Ketefian $\boldsymbol{\nabla}$ Violet H. Barkauskas $\boldsymbol{\nabla}$ Dean G. Smith
}

Background: Nurse staffing levels are an important working condition issue for nurses and believed to be a determinant of the quality of nursing care and patient outcomes.

Objectives: To examine the effects of nurse staffing on adverse events, morbidity, mortality, and medical costs.

Methods: Using two existing databases, the study sample included 232 acute care California hospitals and 124,204 patients in 20 surgical diagnosis-related groups. The adverse events included patient fall/injury, pressure ulcer, adverse drug event, pneumonia, urinary tract infection, wound infection, and sepsis. Multilevel analysis was employed to examine, simultaneously, the effects of nurse staffing and patient and hospital characteristics on patient outcomes.

Results: Three statistically significant relationships were found between nurse staffing and adverse events. An increase of 1 hour worked by registered nurses (RN) per patient day was associated with an $8.9 \%$ decrease in the odds of pneumonia. Similarly, a 10\% increase in RN Proportion was associated with a $9.5 \%$ decrease in the odds of pneumonia. Providing a greater number of nursing hours per patient day was associated with a higher probability of pressure ulcers. The occurrence of each adverse event was associated with a significantly prolonged length of stay and increased medical costs. Patients who had pneumonia, wound infection or sepsis had a greater probability of death during hospitalization.

Conclusion: Patients are experiencing adverse events during hospitalization. Care systems to reduce adverse events and their consequences are needed. Having appropriate nurse staffing is a significant consideration in some cases.

Key Words: adverse events • costs • morbidity • mortality • staffing

$\mathbf{N}$ urse staffing levels are an important working condition issue for nurses and believed to be a determinant of the quality of nursing care and patient outcomes. Recent studies have provided some empirical evidence that nurse staffing influences patient outcomes (ANA, 1997, 2000; Blegen, Goode, \& Reed, 1998; Blegen \& Vaughn, 1998; Kovner \& Gergen, 1998). Patient outcomes examined in these studies focused on adverse events, such as medication errors, pressure ulcers, and postoperative complications. Buerhaus and Needleman (2000) also suggested a set of adverse nurse-sensitive events that included adverse drug events, patient falls and injuries, nosocomial infections, and skin breakdown. Length of stay (LOS) and mortality rates were also examined as nursingsensitive outcomes. However, previous studies were mostly limited to revealing the inverse relationship between nurse staffing and either adverse events, LOS, or mortality, without investigating their relationships thoroughly. This segmented examination may incorrectly infer the causality between staffing and outcomes. For example, several studies have found an inverse relationship between staffing and LOS, suggesting that appropriate nurse staffing could reduce LOS (ANA, 1997, 2000). However, this analytical approach, without taking into account the occurrence of adverse events, does not preclude a competing hypothesis that patients would require more nursing care hours per day as a result of decreases in LOS (Shamian, Hagen, Hu, \& Fogarty, 1994). In addition, few studies examined effects of staffing on medical costs of adverse events.

Among those studies concerning the staffing-outcome relationship, several studies, such as Kovner and Gergen's (1998) recent study, used large public databases. As patient and hospital databases become more available to the public, a growing use of large public databases is expected in the near future. Using large datasets allows comparison of

Sung-Hyun Cho, PhD, MPH, RN, is Chief Researcher, Korea Institute for Health and Social Affairs, Seoul.

Shaké Ketefian, EdD, RN, FAAN, is Professor, University of Michigan School of Nursing, Ann Arbor.

Violet H. Barkauskas, PhD, RN, FAAN, is Associate Professor, University of Michigan School of Nursing, Ann Arbor.

Dean G. Smith, PhD, is Professor, University of Michigan School of Public Health, Ann Arbor. 
patient outcomes across the states or nation, but presents challenges to nurse researchers. Public databases include limited information, especially on nursing staff characteristics and patients' clinical conditions. In addition, a methodological issue in using large datasets is to determine the unit of analysis. Since large datasets include information measured at different levels (e.g., patient, care unit, institution, and geographical region), a decision must be made. A common approach in staffing-outcome research is to aggregate patient-level variables at the institution level. However, this approach may cause incomplete risk adjustment at the patient level, an adjustment that is essential when comparing patient outcomes across institutions. Thus, alternative analytic approaches are needed to examine the relationship between nurse staffing and patient outcomes.

The purpose of this study was to examine the relationship between nurse staffing and patient outcomes with two specific aims: (a) to examine the effects of nurse staffing on adverse events, and (b) to assess the effects of adverse events on morbidity, mortality, and medical costs. This study used large public databases and employed multilevel analysis as an analytic strategy. Multilevel analysis was chosen to examine the staffing-outcome relationship at the individual patient level with the patient as the unit of analysis, and to minimize data aggregation at the institutional level. This study was also designed on the basis of nurse staffing and patient outcomes model (NSPOM) proposed by Cho (2001). The NSPOM provides theoretical explanations of how nurse staffing affects patient outcomes. This model guided the study to thoroughly investigate the effects of nurse staffing on patient outcomes and enhance causality in the staffing-outcome relationship. The effects of nurse staffing on patient outcomes were examined with controlled patient and hospital characteristics. The NSPOM also hypothesizes that there are no direct effects of nurse staffing on morbidity, mortality, and costs. This hypothesis suggests that nurse staffing indirectly influences these three outcomes through adverse events.

\section{Methods}

\section{Data Source}

This study used two existing databases: Hospital Financial Data produced by California's Office of Statewide Health Planning and Development (OSHPD), and the State Inpatient Databases (SID) California-1997 released by the Agency for Healthcare Research and Quality (AHRQ). Hospital Financial Data provided hospital characteristics, nurse staffing, and other financial information. Since hospitals had different reporting periods, databases from three fiscal years (1996-1997, 1997-1998, and 1998-1999) were needed to estimate nursing hours provided and patient days in 1997. The 1998-1999 fiscal year database was used because some hospitals reported nursing hours and patient days provided during 1997 calendar year to this database. The SID California 1997 (the most recent database publicly available at the time this study was proposed) included information of inpatients who were discharged from California hospitals between January 1 and December 31, 1997.

\section{Sample}

The selection of hospitals and patients strived to create a sample that included homogenous hospital and patient groups while representing the majority of the target population. The study sample included 232 acute care hospitals in California, excluding government, long-term care, and "noncomparable" hospitals defined by OSHPD. Further, 20 common surgical diagnosis-related groups (DRGs) were selected as the patient groups (Table 1). Compared to other DRGs, these DRGs had relatively large numerators as well as denominators of adverse event rates, and their major diagnostic categories were not primarily associated with adverse events. The final study sample consisted of 124,204 patients.

\section{Measures}

Hospital characteristics. Ownership, hospital size, teaching affiliation, and location were used as hospital characteristics. Ownership was based on the categories of "Type of Control" defined by the OSHPD (OSHPD, 1998). Nonprofit hospitals included hospitals operated by a church, nonprofit corporation, or nonprofit other. Investor-owned hospitals included hospitals operated by an investor-individual, investor-partnership, or investor-corporation. Hospital size was divided into three groups based on the number of licensed beds stated on the hospital license at the end of the reporting period. Hospitals with 1-99 beds were categorized as small, 100-299 beds as medium, and 300 and more as large. Teaching hospitals indicated those generally recognized as teaching hospitals by the OSHPD. Thus, hospitals not considered teaching hospitals were categorized into nonteaching hospitals in this study. With regard to location, rural hospitals included those designated by the California Rural Health Policy Council as rural (California Rural Health Policy Council, 1998). The other hospitals were defined as nonrural. Based on the four hospital characteristics, four major hospital groups were identified. These hospital groups were used in the comparison of nurse staffing and patient outcomes among hospitals.

Nurse staffing. Nurse staffing in three categories of care units (i.e., medical/surgical acute care, medical/surgical intensive care, and coronary care) were summed and treated as the nurse staffing level of the hospital. Three nurse staffing measures were used to quantify nurse staffing: All Hours, RN Hours, and RN Proportion. All Hours indicated the total productive hours worked by all nursing personnel per patient day. The RN Hours indicated the total productive hours by registered nurses per 
ABLE I. Descriptives of Adverse Events, Length of Stay, Mortality, and Costs by Diagnosis-Related Groups (N = I 24,204)

\begin{tabular}{|c|c|c|c|c|c|c|c|c|c|c|}
\hline \multirow[b]{2}{*}{ DRG category } & \multicolumn{7}{|c|}{ Adverse event rate (\%) } & \multirow[b]{2}{*}{$\begin{array}{l}\text { LOS } \\
\text { (days) }\end{array}$} & \multirow[b]{2}{*}{$\begin{array}{c}\text { Mortality } \\
(\%)\end{array}$} & \multirow[b]{2}{*}{$\begin{array}{l}\text { Costs } \\
\text { (dollars) }\end{array}$} \\
\hline & $\begin{array}{l}\text { Patient P } \\
\text { Fall/Injury }\end{array}$ & $\begin{array}{l}\text { ressure } \\
\text { Ulcer }\end{array}$ & ADE & Pneumonia & UTI & $\begin{array}{l}\text { Wound } \\
\text { Infection }\end{array}$ & Sepsis & & & \\
\hline 1. DRG 1, 2 (Craniotomy) & .26 & .33 & .68 & 4.33 & 3.06 & .49 & .78 & $8.2 \pm 8.1$ & 10.48 & $19,806 \pm 18,975$ \\
\hline 2. DRG 104, 105 (Cardiac valve procedures) & .23 & .26 & 1.48 & 3.27 & 2.15 & 1.08 & 1.25 & $10.0 \pm 7.3$ & 5.34 & $36,209 \pm 21,460$ \\
\hline 3. DRG 106, 107 (Coronary bypass) & .13 & .16 & 1.03 & 2.34 & 1.71 & .96 & .74 & $8.2 \pm 4.9$ & 2.77 & $27,364 \pm 13,367$ \\
\hline 4. DRG 110, 111 (Major cardiovascular procedures) & .26 & .46 & 1.62 & 5.44 & 2.14 & 1.28 & 1.99 & $8.2 \pm 6.9$ & 12.65 & $21,858 \pm 19,074$ \\
\hline 5. DRG 113 (Amputation for circulatory disorders) & .21 & .88 & 1.39 & 3.00 & 2.49 & .91 & 1.94 & $9.9 \pm 8.5$ & 5.55 & $14,242 \pm 15,015$ \\
\hline 6. DRG 146, 147 (Rectal resection) & .27 & .20 & 1.00 & 1.26 & 1.93 & 2.73 & 1.00 & $8.2 \pm 4.6$ & 1.60 & $12,503 \pm 8,223$ \\
\hline 7. DRG 148, 149 (Major small and large bowel procedures) & .18 & .28 & 1.11 & 3.33 & 1.94 & 3.67 & 1.82 & $9.9 \pm 8.0$ & 4.95 & $16,494 \pm 17,036$ \\
\hline 8. DRG 154, 155 (Stomach, esophageal, and duodenal procedures) & .07 & .18 & .98 & 6.01 & 2.13 & 3.23 & 2.45 & $9.6 \pm 9.0$ & 7.07 & $18,834 \pm 21,180$ \\
\hline 9. DRG 191, 192 (Pancreas, liver, and shunt procedures) & .20 & .30 & .90 & 4.39 & 1.90 & 3.69 & 3.99 & $12.0 \pm 11.9$ & 7.93 & $25,296 \pm 28,024$ \\
\hline 10. DRG 209 (Major joint and limb reattachment) & .25 & .17 & .89 & .93 & 1.26 & .26 & .15 & $4.7 \pm 2.6$ & .69 & $11,496 \pm 5,208$ \\
\hline 11. DRG 210, 211 (Hip and femur procedures) & .26 & .33 & 1.22 & 1.81 & 2.27 & .26 & .45 & $5.6 \pm 3.6$ & 1.66 & $9,278 \pm 6,374$ \\
\hline Overall & .21 & .26 & 1.06 & 2.59 & 1.87 & 1.25 & .97 & $7.4 \pm 6.3$ & 3.92 & $17,899 \pm 15,908$ \\
\hline
\end{tabular}

Note. $\mathrm{DRG}=$ diagnosis-related groups; $\mathrm{ADE}=$ adverse drug event; $\mathrm{UTI}=$ urinary tract infection; $\mathrm{LOS}=$ length of stay. 
patient day. The RN Proportion referred to "skill mix" of nursing hours, calculated as RN Hours divided by All Hours.

Patient characteristics. Patient characteristics consisted of (a) age, (b) sex, (c) race, (d) primary payer, (e) DRG, (f) number of diagnoses at admission, and (g) type of admission (scheduled or unscheduled). Some DRGs were collapsed into a category (e.g., "rectal resection without complications and/or comorbidity (CC)" and "rectal resection with CC"). This regrouping was necessary because of low incidences of adverse events for each DRG. The 20 DRGs were regrouped into $11 \mathrm{DRG}$ categories. The number of diagnoses was computed by counting all nonmissing principal and secondary diagnoses that were already present at the time of admission. Diagnoses that were present at admission were used to enable the variable "number of diagnoses" to reflect the severity and comorbidity of a patient at the time of admission, not at the time of discharge. The SID California defined a "scheduled admission" as scheduled at least 24 hours before admission (AHRQ, 2000).

Adverse events. This study included seven adverse events: (a) patient fall/injury, (b) pressure ulcer, (c) adverse drug event (ADE), (d) pneumonia, (e) urinary tract infection (UTI), (f) wound infection, and (g) sepsis. Adverse events were detected by using the International Classification of Diseases, 9th Revision, Clinical Modification (ICD9-CM) diagnosis codes. The ICD-9-CM diagnosis codes associated with adverse events were principally identified from the literature. Further, the list of the ICD-9 codes was examined by an expert panel for validity. A questionnaire was sent to 15 master-prepared clinical nurse specialists or nurse practitioners who were currently practicing at a healthcare facility. The first question addressed the preventability of adverse events by adequate nurse staffing; and the second question asked the minimization of the consequences of adverse events by adequate nurse staffing. The expert panel was asked to answer with a range of 1 to 5: 1 (definitely not), 2 (unlikely), 3 (possibly), 4 (probably), and 5 (definitely). Seven of the 15 nurses completed and returned the questionnaire, a $47 \%$ response rate. The ICD9-CM diagnosis codes that had an average of 3 or higher from either question were considered nursing-sensitive adverse events and included in the analysis.

The SID California 1997 included a new variable, "time of onset," which indicated whether a diagnosis was present at admission. This information was expected to decrease the effects of underlying disease process on adverse events and consequently strengthen the causality between nurse staffing and the occurrence of adverse events. This study included adverse events only when they were not present at admission.

Morbidity and mortality. The impact of adverse events on morbidity was indirectly measured by LOS. The LOS indicated the number of days that the patient spent in the hospital, including the day of admission, but not the day of discharge. The SID assigned "zero" of LOS for a patient whose admission and discharge date are the same. This study treated "zero" LOS as a one-day stay $($ LOS $=1$ ). Mortality was measured as dichotomous; died, or did not die during hospitalization.

Costs. Because there isn't necessarily a relationship between cost and charges (Finkler, 1982), charges were converted to costs by using hospital-level ratios of costs-tocharges. Using OSHPD data, the cost-to-charge ratio was calculated as total operating expenses divided by gross patient revenue. Costs for individual patients were estimated by multiplying charges and the hospital-specific cost-to-charge ratio. Due to high skewness of LOS and costs, both were transformed to their natural logarithmic value for statistical analysis.

\section{Data Analysis}

Descriptive analyses on patient and hospital characteristics were conducted before statistical analyses. Analyses to answer the research questions for study aims employed multilevel regression models instead of conventional linear or logistic regression. The multilevel models assumed that the data had two levels of data structure (Level $1=$ patient level; Level 2 = hospital level). This multilevel analysis allowed simultaneous examination of the effects of nurse staffing, and patient and hospital characteristics on patient outcomes. Multilevel analysis was employed using SAS PROC MIXED for continuous dependent variables, and

TABLE 2. Adjusted Odds Ratio $(95 \% \mathrm{Cl})$ of Nurse Staffing on Adverse Events $(\mathbf{N}=123,095)$

\begin{tabular}{lccc} 
& All Hours & RN Hours & RN Proportion \\
\hline Patient fall/injury & $1.08(.99-1.18)$ & $1.07(.96-1.19)$ & $.96(.21-4.49)$ \\
Pressure ulcer & $1.13(1.01-1.27)^{\star}$ & $1.11(.97-1.27)$ & $.75(.11-4.98)$ \\
ADE & $1.04(.96-1.13)$ & $1.01(.92-1.11)$ & $.62(.16-2.38)$ \\
Pneumonia & $.96(.91-1.01)$ & $.91(.85-.97)^{\star *}$ & $.37(.15-.91)^{\star}$ \\
UTI & $1.02(.95-1.08)$ & $1.01(.93-1.08)$ & $.92(.31-2.64)$ \\
Wound infection & $1.00(.95-1.06)$ & $.97(.91-1.04)$ & $.52(.21-1.30)$ \\
Sepsis & $1.01(.95-1.08)$ & $1.02(.95-1.09)$ & $1.20(.43-3.33)$ \\
\hline
\end{tabular}

Note. $\mathrm{ADE}=$ Adverse drug event; $\mathrm{UTI}=$ Urinary tract infection .

${ }^{*} p<.05 ;{ }^{* *} p<.01$. 
GLIMMIX SAS macro for dichotomous outcomes (Littell, Milliken, Stroup, \& Wolfinger, 1996).

\section{Results}

The mean All Hours of 232 hospitals was 8.9 hours per patient day. On average, patients were provided with 6.3 hours of RN staffing per patient day (RN Hours). Seventy-one percent of All Hours were provided by RNs (RN Proportion). Results from descriptive analyses of adverse events, morbidity, mortality, and costs are presented in Table 1 . As expected, adverse events rarely occurred, with the majority of patients $(93.2 \%)$ having no adverse events. Out of 124,204 patients, 6,982 (5.6\%) experienced only one of the defined seven adverse events. About $1.2 \%$ $(1,461)$ patients had more than one adverse event with the maximum being four events. This finding indicates that $17.3 \%$ of patients who experienced at least one adverse event had two or more adverse events during hospitalization. Pneumonia occurred most frequently $(2.59 \%)$ among the seven adverse events, whereas falls/injuries had the lowest rate of occurrence. Adverse event rates varied among the 11 DRG categories and were statistically different. Patients who had amputations (DRG 113) had the highest rate of pressure ulcers. Wound infections occurred most frequently in patients with disorders of the digestive or hepatobiliary system. Great variations in LOS, mortality, and costs were also found among DRGs. Those who had pancreas, liver, and shunt procedures (DRGs 191 and 192) had the longest average LOS (12.0 days) whereas patients with major joint and limb reattachment procedures of lower extremity (DRG 209) had the shortest average LOS (4.7 days). Major cardiovascular procedures (DRGs 110 and 111) had the highest mortality rates. Cardiac valve procedures, (i.e., the reference group) were the most expensive procedures. Hip and femur procedures incurred the lowest costs.

\section{Effects of Nurse Staffing on Adverse Events}

To isolate effects of nurse staffing on adverse events, analyses controlled for patient and hospital characteristics. Patient characteristics were significantly associated with adverse events. Age had a significantly positive relationship with most adverse events except patient fall/injury. Male patients were more likely to have pressure ulcers, ADEs, pneumonia, wound infections, and sepsis. Female patients had a higher probability of UTI than males. Wound infection and sepsis occurred more frequently in Black and Hispanic patients as compared to Whites. Primary payer also had a significant relationship with all adverse events except fall/injury and pressure ulcer. Patients whose primary payer was private insurance had a lower probability of having adverse events than Medicare patients, controlling for other patient characteristics. The probability of all adverse events except fall/injury significantly differed among DRGs. Patients whose admission was unscheduled were more likely to have an adverse event excluding fall/injury. A greater number of diagnoses at admission, which reflected patient's comorbidity, were associated with having adverse events except wound infection. These findings suggest that patient characteristics used in this study pro- vided good indicators for risk adjustment for adverse events.

Hospital characteristics were also significantly related to adverse events. Patients treated in investor-owned hospitals had a higher tendency to have UTIs and sepsis. With respect to hospital size, three types of nosocomial infections (UTIs, wound infections, and sepsis) occurred most frequently in large hospitals.

Finally, the odds ratios (ORs) that adjusted for all patient and hospital factors were produced to examine the relationship between nurse staffing and adverse events (Table 2). Among 21 multilevel logistic regression models, there were three statistically significant relationships. An unexpected relationship was found that All Hours had a positive relationship with pressure ulcers $(\mathrm{OR}=1.13)$. More in line with expectations, RN Hours and RN Proportion had a significant inverse relationship with pneumonia. An increase of $1 \mathrm{RN}$ Hour was associated with a decrease of $8.9 \%(\mathrm{OR}=0.911)$ in the odds of pneumonia. This estimation indicates that, on the average, the probability of pneumonia is $0.23 \%$ lower for 1 -hour increase in RN Hours, decreasing the overall pneumonia rate from $2.59 \%$ to $2.36 \%$. The odds ratio of RN Proportion on pneumonia $(\mathrm{OR}=0.3686)$ in Table 2 indicates the effect of $100 \%$ increase of RN Proportion. Using this estimate, an increase of $10 \%$ in RN Proportion corresponded to a decrease of $9.5 \%(\mathrm{OR}=0.905)$ in the odds of pneumonia.

To provide parsimonious summaries from the multilevel logistic regression, the probability of pneumonia for an average patient was estimated for four hospital groups (Groups A-D) that were categorized by hospital ownership, size, location, and teaching affiliation. These hospital groups were the most common and accounted for $81 \%$ of 232 hospitals included in this study. An average patient was defined as (a) a 68-year-old White woman with a scheduled admission with five diagnoses, (b) having a cardiac valve procedure, and (c) using Medicare as the primary payer. The estimated probabilities of pneumonia varied among hospital groups and nurse staffing levels (Table 3). For example, when 6 RN Hours were provided per patient day, the probability that an average patient would have pneumonia ranged from a low of $1.59 \%$ in Group B to a high of $1.81 \%$ in Group A. Further, an increase of one RN hour per patient day corresponded to a $0.12-0.19 \%$ decrease in the probability of pneumonia. For example, when RN hours increase from 6 to 7 hours per patient day, the probability of pneumonia in a patient in Group B would be decreased by $0.14 \%$, which indicates an $8.8 \%(0.14 / 1.59)$ decrease in the probability of pneumonia. In addition, with RN Proportion of $70 \%$ nursing hours provided, the probability of pneumonia for each hospital group ranged from $1.56 \%$ to $1.78 \%$. Hospitals in Group D had the highest probability of pneumonia, whereas Group B had the lowest. An increase of $10 \%$ in RN Proportion was related to a decrease of about $0.14-0.20 \%$ in the probability of pneumonia. When RN Proportion increases from $70 \%$ to $80 \%$, the probability of a patient in Group B would be decreased by $0.14 \%$, which indicates a $9.0 \%(0.14 / 1.56)$ decrease in the probability of pneumonia. 
TABLE 3. Estimated Probability of Pneumonia (\%) for an Average Patient by Hospital Groups

\begin{tabular}{|c|c|c|c|c|c|c|c|c|c|c|}
\hline & \multicolumn{5}{|c|}{ RN Hours (hours/patient day) } & \multicolumn{5}{|c|}{ RN Proportion (\%) } \\
\hline & 4 & 5 & 6 & 7 & 8 & 50 & 60 & 70 & 80 & 90 \\
\hline Group A $(n=12)$ & 2.17 & 1.98 & 1.81 & 1.65 & 1.51 & 2.08 & 1.89 & 1.71 & 1.55 & 1.41 \\
\hline \multicolumn{11}{|l|}{ Large, nonprofit, teaching, nonrural } \\
\hline Group B $(n=79)$ & 1.91 & 1.75 & 1.59 & 1.45 & 1.33 & 1.90 & 1.72 & 1.56 & 1.42 & 1.28 \\
\hline \multicolumn{11}{|l|}{ Medium, nonprofit, nonteaching, nonrural } \\
\hline Group C $(n=48)$ & 2.06 & 1.88 & 1.72 & 1.57 & 1.43 & 2.03 & 1.84 & 1.67 & 1.51 & 1.37 \\
\hline \multicolumn{11}{|l|}{ Large, nonprofit, nonteaching, nonrural } \\
\hline Group D $(n=48)$ & 2.09 & 1.91 & 1.74 & 1.59 & 1.45 & 2.16 & 1.96 & 1.78 & 1.61 & 1.46 \\
\hline Medium, investor-owned, nonteaching, nonrural & & & & & & & & & & \\
\hline
\end{tabular}

Effects of Adverse Events on Morbidity, Mortality, and Costs The occurrence of all adverse events was associated with a significantly prolonged LOS. Pressure ulcers (regression coefficient $=0.6086$ ) had the greatest impact on LOS, followed by pneumonia and wound infections (Table 4). The occurrence of a pressure ulcer was associated with a 1.84fold increase in LOS. Using the grand mean of LOS $(7.4$ days) on average, having pressure ulcer would increase LOS by 6.2 days. Pneumonia was associated with a 1.74 fold increase in LOS. All patient characteristics had a statistically significant relationship with LOS, whereas no hospital characteristics influenced LOS.

Adverse events were also associated with increased mortality. Pneumonia, wound infection, and sepsis were positively related to increased mortality. Sepsis had the greatest impact on mortality $(\mathrm{OR}=7.40)$. Pneumonia was associated with a 3.39-fold increase in the odds of death. Interestingly, the occurrence of UTI was associated with lower probability of death. All patient characteristics except sex and race had a significant relationship with mortality. Patients whose primary payer was categorized as "other," including self-pay and no charge, had the highest probability of death, followed by Medicaid patients. Unscheduled admission and a greater number of diagnoses were associated with a higher probability of death. Patients from large hospitals had a statistically lower probability of death than patients from other hospitals.

All adverse events were associated with increased costs. Sepsis had the greatest increase in costs, followed by pneumonia. Suffering pneumonia was associated with a 1.84 fold increase in costs. All patient characteristics also influenced medical costs. While advanced age had a positive relationship with LOS and mortality, age had a negative relationship with costs. Patients whose primary payer was private insurance had the shortest LOS and incurred the least costs whereas patients with Medicaid had the longest LOS and greatest costs. Teaching hospitals had higher costs than nonteaching hospitals.

Table 5 compares the estimated LOS, mortality, and costs for an average patient with and without pneumonia. The occurrence of pneumonia was associated with an increase of 5.1-5.4 days in LOS, $4.67-5.55 \%$ in the probability of death, and \$22,390-28,505 in costs. For example, when a patient in Group B suffered pneumonia during hospitalization, her/his LOS would increase by 5.2 days, which indicates a $75 \%(5.2 / 6.9)$ increase in LOS. Having pneumonia was also associated with a $220 \%(5.55 / 2.52)$ increase in the probability of death, and an $84 \%$ $(22,860 / 27,362)$ increase in costs.

\section{Discussion}

A major finding in this study was the great impact of patient characteristics on the occurrence of adverse events, while hospital characteristics had minimal influence. After controlling for patient and hospital characteristics, RN staffing (RN Hours and RN Proportion) exhibited the expected inverse relationship with pneumonia. The strong relationship between RN staffing and pneumonia can be attributed to the heavy responsibility of RNs in lung care for surgical patients. Postoperative patients are at particularly high risk of pneumonia due to atelectasis, retained secretions, and pain (Gaynes, 1998). Attentive lung care provided by RNs may allow surgical patients to avoid postoperative pulmonary infections. Adequate RN staffing would result in good infection control practice, permitting them to adhere to aseptic techniques and standard care. Because these principles and techniques require a high level of knowledge and skill, RN staffing would be more important in preventing pneumonia than overall nurse staffing.

Contrary to the assumption that frequent position changes provided by adequate staffing can prevent pressure ulcers, All Hours had a positive relationship with pressure ulcers. Blegen, Goode, and Reed (1998) also reported that higher total nursing hours were associated with higher rates of decubiti, suggesting that units with high patient acuity provided high nursing hours and had high rates of decubiti. The positive relationship may be attributed to incomplete risk adjustment that would omit important risk factors. Further studies need to add risk factors specific to pressure ulcers in surgical patients, such as immobility, malnutrition, operating time, and conditions on the operating table (Bliss \& Simini, 1999; Kemp, Keithley, Smith, \& Morreale, 1990), to isolate the effects of nurse staffing on pressure ulcers from those of patient risk factors. Given the simultaneity permitted in the model employed, this unusual result was not expected. However, a possible explanation would be that higher staffing level 
TABLE 4. Multilevel Regression Summary for Length of Stay, Mortality, and Costs $(N=12$ I, 2 I5)

\begin{tabular}{|c|c|c|c|}
\hline & LOS & Mortality & Costs \\
\hline & Coefficient $(95 \% \mathrm{Cl})$ & OR $(95 \% \mathrm{Cl})$ & Coefficient (95\% Cl) \\
\hline Age & $.0013(.0011, .0015)^{\star *}$ & $1.02(1.02,1.03) * *$ & $-.0014(-.0016,-.0012)^{\star \star}$ \\
\hline Male (vs female) & $-.0164(-.0225,-.0103)^{\star \star}$ & $1.03(.97,1.09)$ & $.0138(.0085, .0191)^{\star \star}$ \\
\hline \multicolumn{4}{|l|}{ Race (vs White) } \\
\hline Black & $.0583(.0432, .0734)^{\star \star}$ & $90(.78,1.03)$ & $.0317(.0188, .0446)^{\star \star}$ \\
\hline Hispanic & $.0256(.0154, .0358)^{\star \star}$ & $1.08(.98,1.18)$ & $.0134(.0046, .0222)^{\star \star}$ \\
\hline Other & $.0311(.0186, .0436)^{\star *}$ & $1.06(.95,1.19)$ & $.0127(.0017, .0237)^{\star}$ \\
\hline \multicolumn{4}{|l|}{ Primary payer (vs Medicare) } \\
\hline Medicaid & $.1013(.0872, .1154)^{\star *}$ & $1.21(1.08,1.37)^{\star *}$ & $.0492(.0370, .0614)^{\star *}$ \\
\hline Private insurance & $-.0393(-.0471,-.0315)^{\star \star}$ & $.93(.86,1.01)$ & $-.0182(-.0249,-.0115)^{\star *}$ \\
\hline Other & $-.0050(-.0211, .0111)$ & $1.26(1.08,1.47)^{\star \star}$ & $.0126(-.0013, .0265)$ \\
\hline \multicolumn{4}{|l|}{ DRG (vs DRG 104, 105) } \\
\hline 1 (DRG 1, 2) & $-.3066(-.3237,-.2895)^{\star \star}$ & $2.25(1.98,2.55)^{\star *}$ & $-.8154(-.8303,-.8005)^{\star *}$ \\
\hline 3 (DRG 106, 107) & $-.1144(-.1285,-.1003)^{\star \star}$ & $.44(.39, .50)^{\star \star}$ & $-.1984(-.2106,-.1862)^{\star *}$ \\
\hline 4 (DRG 110, 111) & $-.3089(-.3265,-.2913)^{\star *}$ & $2.18(1.93,2.47)^{* *}$ & $-.6364(-.6517,-.6211)^{\star *}$ \\
\hline 5 (DRG 113) & $-.2793(-.3011,-.2575)^{\star \star}$ & $.51(.43, .61)^{\star *}$ & $-1.2962(-1.3150,-1.2774)$ \\
\hline 6 (DRG 146, 147) & $.0121(-.0165, .0407)$ & $.37(.25, .55)^{\star \star}$ & $-.9566(-.9815,-.9317)^{\star \star}$ \\
\hline 7 (DRG 148, 149) & $-.0065(-.0212, .0082)$ & $.68(.60, .77)^{\star \star}$ & $-.9385(-.9512,-.9258)^{\star *}$ \\
\hline 8 (DRG 154, 155) & $-.2035(-.2219,-.1851)^{\star \star}$ & $1.04(.90,1.21)$ & $-.9290(-.9451,-.9129)^{\star \star}$ \\
\hline 9 (DRG 191, 192) & $-.0275(-.0534,-.0016)^{\star}$ & $1.17(.96,1.42)$ & $-.7388(-.7611,-.7165)^{\star \star}$ \\
\hline 10 (DRG 209) & $-.4691(-.4832,-.4550)^{\star *}$ & $.16(.13, .19)^{\star *}$ & $-.9198(-.9321,-.9075)^{\star \star}$ \\
\hline 11 (DRG 210, 211) & $-.5792(-.5951,-.5633)^{\star *}$ & $.15(.13, .18)^{\star *}$ & $-1.3690(-1.3827,-1.3553)^{\star *}$ \\
\hline Unscheduled admission & $.2124(.2057, .2191)^{\star *}$ & $3.17(2.95,3.41)^{\star \star}$ & $.1385(.1328, .1442)^{\star *}$ \\
\hline Number of diagnoses & $.0514(.0502, .0526)^{\star *}$ & $1.13(1.12,1.14)^{\star \star}$ & $.0535(.0525, .0545)^{\star \star}$ \\
\hline Investor-owned vs nonprofit & $.0159(-.0239, .0557)$ & $.93(.81,1.06)$ & $-.0284(-.0878, .0310)$ \\
\hline \multicolumn{4}{|l|}{ Size (vs small) } \\
\hline Medium & $.0038(-.0521, .0597)$ & $.91(.72,1.16)$ & $-.0285(-.1110, .0540)$ \\
\hline Large & $-.0104(-.0751, .0543)$ & $.75(.58, .97)^{\star}$ & $-.0386(-.1348, .0576)$ \\
\hline Teaching vs nonteaching & $.0562(-.0212, .1336)$ & $1.12(.90,1.39)$ & $.2310(.1130, .3490)^{\star \star}$ \\
\hline Rural vs nonrural & $-.0092(-.0851, .0667)$ & $.74(.54,1.02)$ & $-.0054(-.1181, .1073)$ \\
\hline Fall/injury & $.2554(.1935, .3173)^{\star *}$ & $.80(.45,1.43)$ & $.2456(.1919, .2993)^{\star \star}$ \\
\hline Pressure ulcer & $.6086(.5525, .6647)^{\star *}$ & $1.26(.89,1.77)$ & $.5304(.4818, .5790)^{\star *}$ \\
\hline ADE & $.2711(.2435, .2987)^{\star *}$ & $.77(.60,1.00)$ & $.2246(.1644, .2848)^{\star *}$ \\
\hline Pneumonia & $.5545(.5363, .5727)^{\star *}$ & $3.39(3.08,3.73)^{\star *}$ & $.6073(.5914, .6232)^{\star *}$ \\
\hline UTI & $.4309(.4097, .4521)^{\star \star}$ & $.81(.69, .96)^{\star}$ & $.3187(.3001, .3373)^{\star \star}$ \\
\hline Wound infection & $.5465(.5202, .5728)^{\star *}$ & $1.29(1.09,1.52)^{\star \star}$ & $.5073(.4844, .5302)^{\star *}$ \\
\hline Sepsis & $.4994(.4692, .5296)^{\star *}$ & $7.40(6.46,8.48)^{\star *}$ & $.6923(.6660, .7186)^{\star \star}$ \\
\hline
\end{tabular}

Note. $\mathrm{DRG}=$ diagnosis-related groups; $\mathrm{ADE}=$ adverse drug event; $\mathrm{UTI}=$ urinary tract infection; ${ }^{*} p<.05 .{ }^{* *} p<.01$.

might enable nurses to assess skin integrity more frequently and consequently detect more pressure ulcers.

This study reported lower adverse event rates as compared to previous studies. For example, Thomas et al. (2000) reported a fall rate of $1.5 \%$ and fracture-related rate of $0.4 \%$, while this study had a fall/injury rate of
$0.21 \%$. In addition, the UTI rate $(1.87 \%)$ in this study is also lower than a UTI rate of $3.58 \%$ reported by Kovener and Gergen's (1998) study that included patients who had major surgery. Low rates of adverse events may be attributed to several factors. First, this study used the variable "time of onset" that indicated whether primary and sec- 
TABLE 5. Estimated LOS, Mortality, and Costs for an Average Patient by Hospital Groups

\begin{tabular}{|c|c|c|c|c|c|c|}
\hline & \multicolumn{2}{|c|}{ LOS (days) } & \multicolumn{2}{|c|}{ Mortality (\%) } & \multicolumn{2}{|c|}{ Costs (dollars) } \\
\hline & $\begin{array}{c}\text { Without } \\
\text { Pneumonia }\end{array}$ & $\begin{array}{c}\text { With } \\
\text { Pneumonia }\end{array}$ & $\begin{array}{c}\text { Without } \\
\text { Pneumonia }\end{array}$ & $\begin{array}{c}\text { With } \\
\text { Pneumonia }\end{array}$ & $\begin{array}{c}\text { Without } \\
\text { Pneumonia }\end{array}$ & $\begin{array}{c}\text { With } \\
\text { Pneumonia }\end{array}$ \\
\hline $\begin{array}{l}\text { Group A ( } n=12) \\
\text { Large, nonprofit, teaching, nonrural }\end{array}$ & 7.2 & 12.6 & 2.34 & 7.50 & 34,125 & 62,630 \\
\hline $\begin{array}{l}\text { Group B ( } n=79 \text { ) } \\
\text { Medium, nonprofit, nonteaching, nonrural }\end{array}$ & 6.9 & 12.1 & 2.52 & 8.07 & 27,362 & 50,222 \\
\hline $\begin{array}{l}\text { Group C ( } n=48) \\
\quad \text { Large, nonprofit, nonteaching, nonrural }\end{array}$ & 6.8 & 11.9 & 2.09 & 6.76 & 27,087 & 49,717 \\
\hline $\begin{array}{l}\text { Group D }(n=48) \\
\text { Medium, investor-owned, nonteaching, nonrural }\end{array}$ & 7.0 & 12.3 & 2.34 & 7.52 & 26,798 & 49,188 \\
\hline
\end{tabular}

Note. LOS = Length of stay.

ondary diagnoses were present at the time of admission. While previous studies included adverse events in secondary diagnoses, this study considered diagnoses an adverse event only if these diagnoses were not present at admission. Excluding diagnoses present at admission did cause low incidence rates of adverse events as compared to other studies, yet strengthened the causality between nurse staffing and adverse events.

Second, this study used ICD-9 diagnosis codes to detect adverse events, while some previous studies used incidence reports or other methods (e.g., patient record review, observation). Regardless of the differences in definitions of adverse events among studies, the use of different methods of data collection could influence the observed incidence of adverse events. In particular, use of ICD-9 codes may cause underreporting and consequently lower incidence rates of adverse events than actually occurred. Another reason for low incidence rates could be that medical patients were excluded in this study, who are likely to be sicker than surgical patients. While surgical patients are expected to be healthy enough to have surgery, medical patients may be more susceptible to adverse events. For example, there would be more bed-ridden patients among medical patients than surgical patients, which increases the risk of pressure ulcers.

This study also revealed the great impact of adverse events on LOS, mortality, and costs. However, this impact may be overestimated. For example, while this study found that ADEs increased about 2.3 days in LOS and $\$ 4,500$ in costs, a matched case-control study of ADEs reported 1.74 days and $\$ 2,013$ as the excess LOS and costs attributable to ADEs (Classen, Pestotnik, Evans, Lloyd, \& Burke, 1997). Although multivariate analysis conducted in this study controlled for patient characteristics, it may not isolate the unique effect of a certain adverse event on LOS, morbidity, and costs. Further studies need to use more precise research designs to produce more accurate estimates of excess LOS, death, and costs.

Several limitations were identified in this study. The first relates to measurement issues regarding nurse staffing.
Aggregated nurse staffing measures may have smoothed the level of staffing over the year, thus did not account for the variability in either patient census or in nursing hours. Given the fluctuations in both nurse staffing and patient census, even a hospital that had a high staffing level would have times during the year when it was staffed better or worse than at other times. Because of this measurement issue, estimates from the statistical analyses could underestimate the effects of nurse staffing on patient outcomes. In addition, this study focused on quantifying nurse staffing levels, while professional characteristics of nursing personnel (e.g., experience, educational preparation, and certification) that also influence patient outcomes were not considered. Second, this study did not investigate organizational characteristics of hospitals. Only four hospital characteristics were included, although organizational traits and work environments affect both nurse staffing and patient outcomes (Aiken \& Patrician, 2000). Third, the effects of nurse staffing on patient outcomes could not be completely captured. Nurse staffing is expected to influence other dimensions of patient outcomes, in particular, positive patient outcomes. In addition, the seven adverse events used in this study are not exhaustive. Other types of complications may, in some circumstances, be attributed to inadequate nurse staffing.

A lack of statistical significance on some results should not overshadow the possible effects of nurse staffing on patient outcomes. Nurse staffing, especially RN staffing, showed inverse relationships with several adverse events although they did not reach statistical significance. This study also indicates that patients are experiencing adverse events during hospitalization. Nurses, as patient advocates, are responsible for protecting patients from adverse outcomes, especially those patients at a higher risk of adverse events. Findings from this study also suggest that hospitals could reduce operational costs by preventing adverse events.

Future studies need to evaluate appropriateness of ICD-9-CM codes in examining nursing care quality. Geraci, Ashton, Kuykendall, Johnson, and Wu (1997) concluded that ICD-9-CM codes are poor measures of com- 
plication occurrence. Nurse researchers may test the ability of ICD-9-CM codes in patient discharge databases to detect adverse events that actually occurred by using a prospective study or medical record review. Furthermore, ICD-9 diagnosis and procedure codes that are associated with nursing care need to be explored. This study used an expert panel to evaluate which diagnosis codes could be attributed to nursing care. However, this review was not extensive or thorough. Further studies are required to explore ICD-9 diagnosis codes and develop a complete list of codes related to nursing care quality.

Finally, evaluating the process of nursing care still remains key to understanding the relationship between nurse staffing and patient outcomes. Various approaches to conceptualizing this relationship and producing empirical findings are necessary to explain the structure-outcome relationship and strengthen its causality.

Accepted for publication September 16, 2002.

This project was supported by grant number R03 HS11397 from the Agency for Healthcare Research and Quality.

The authors thank Dr. Edward Rothman, Professor of Statistics and Director, and Ms. Kathy Welch, Statistical Consultant, at the Center for Statistical Consultation and Research, University of Michigan, for their assistance in the statistical analysis.

Corresponding author: Sung-Hyun Cho, Korea Institute for Health and Social Affairs, Department of Health Policy, San 4214 Bulgwang-dong Eunpyeong-gu Seoul, Korea 122-705 (e-mail: shcho@kihasa.re.kr).

\section{References}

Agency for Healthcare Research and Quality. (2000). Healthcare cost and utilization project state inpatient databases. Rockville, MD: Agency for Healthcare Research and Quality.

Aiken, L. H., \& Patrician, P. A. (2000). Measuring organizational traits of hospitals: The revised nursing work index. Nursing Research, 49(3), 146-153.

American Nurses Association. (1997). Implementing nursing's report card: A study of $R N$ staffing, length of stay and patient outcomes. Washington, DC: American Nurses Publishing.

American Nurses Association. (2000). Nurse staffing and patient outcomes in the inpatient hospital setting. Washington, DC: American Nurses Publishing.

Blegen, M. A., Goode, C. J., \& Reed, L. (1998). Nurse staffing and patient outcomes. Nursing Research, 47(1), 43-50.
Blegen, M. A., \& Vaughn, T. (1998). A multisite study of nurse staffing and patient occurrences. Nursing Economic\$, 16(4), 196-203.

Bliss, M., \& Simini, B. (1999). When are the seeds of postoperative pressure sores sown?: Often during surgery. BMJ, 319, 863-864.

Buerhaus, P. I., \& Needleman, J. (2000). Policy implications of research on nurse staffing and quality of patient care. Policy, Politics, \& Nursing Practice, 1(1), 5-15.

California Rural Health Policy Council. (1998). California general acute care hospitals in rural and nonrural areas selected utilization and financial data 1996 and 1997. Sacramento, CA: California Rural Health Policy Council.

Cho, S. H. (2001). Nurse staffing and adverse patient outcomes: A systems approach. Nursing Outlook, 49(2), 78-85.

Classen, D. C., Pestotnik, S. L., Evans, R. S., Lloyd, J. F., \& Burke, J. P. (1997). Adverse drug events in hospitalized patients: Excess length of stay, extra costs, and attributable mortality. JAMA, 277(4), 301-306.

Finkler, S. A. (1982). The distinction between cost and charges. Annals of Internal Medicine, 96, 102-109.

Gaynes, R. P. (1998). Surveillance of nosocomial infections. In J. V. Bennett \& P. S. Brachman (Eds.), Hospital Infections (4th ed., pp. 65-84). Philadelphia: Lippincott.

Geraci, J. M., Ashton, C. M., Kuykendall, D. H., Johnson, M. L., \& Wu, L. (1997). International Classification of Diseases, 9th Revision, Clinical Modification Codes in discharge abstracts are poor measures of complication occurrence in medical inpatients. Medical Care, 35(6), 589-602.

Kemp, M., Keithley, J. K., Smith, D. W., \& Morreale, B. (1990). Factors that contribute to pressure sore in surgical patients. Research in Nursing \& Health, 13, 293-301.

Kovner, C., \& Gergen, P. J. (1998). Nurse staffing levels and adverse events following surgery in U.S. hospitals. Image: Journal of Nursing Scholarship, 30(4), 315-321.

Littell, R. C., Milliken, G. A., Stroup, W. W., \& Wolfinger, R. D. (1996). SAS system for mixed models. Cary, NC: SAS Institute.

Office of Statewide Health Planning and Development. (1998). Hospital Annual Financial Data: Selected Datafile Documentation for Report Periods Ended January 1, 1997 through December 31, 1997. Sacramento, CA: State of California Office of Statewide Health Planning and Development.

Shamian, J., Hagen, B., Hu, T. W., \& Fogarty, T. E. (1994). The relationship between length of stay and required nursing care hours. Journal of Nursing Administration, 24(7,8), 52-58.

Thomas, E. J., Studdert, D. M., Burstin, H. R., Orav, E. J., Zeena, T., Williams, E. J., et al. (2000). Incidence and types of adverse events and negligent care in Utah and Colorado. Medical Care, 38(3), 261-271. 